\title{
An introduction to neutron reflectometry
}

\author{
Fabrice Cousin ${ }^{1, *}$ and Giulia Fadda ${ }^{1,2}$ \\ ${ }^{1}$ Université Paris-Saclay, Laboratoire Léon-Brillouin, CEA-CNRS, CEA-Saclay, F-91191 Gif-sur- \\ Yvette, France \\ ${ }^{2}$ Université Sorbonne Paris Nord, UFR SMBH, 74 rue Marcel-Cachin, F-93017 Bobigny, France
}

\begin{abstract}
Specular neutron reflectivity is a neutron diffraction technique that provides information about the structure of surfaces or thin films. It enables the measurement of the neutron scattering length density profile perpendicular to the plane of a surface or an interface, and thereby gives access to the profile of the chemical composition of the film. The wave-particle duality allows to describe neutrons as waves; at an interface between two media of different refractive indexes, neutrons are partially reflected and refracted by the interface. Interferences can occur between waves reflected at the top and at the bottom of a thin film at an interface, which gives rise to interference fringes in the reflectivity profile directly related to its thickness. The characteristic sizes that can be probed range from $5 \AA$ to $2000 \AA$. Neutron-matter interaction directly occurs between neutron and the atom nuclei, which enable to tune the contrast by isotopic substitution. This makes it particularly interesting in the fields of soft matter and biophysics. This course is composed of two parts describing respectively its principle and the experimental aspects of the method (instruments, samples). Examples of applications of neutron reflectometry in the biological domain are presented by Y. Gerelli in the book section "Applications of neutron reflectometry in biology".
\end{abstract}

\section{Introduction}

Neutron reflectometry (NR) is a surface technique that enables to measure the thickness and the chemical composition of one or several thin layers at a surface or an interface. Typical neutron wavelengths used during experiments range from $3 \AA$ to $25 \AA$, which allows to study thicknesses from $5 \AA$ to $2000 \AA$. A neutron beam is sent, at a grazing angle, to the interface or the surface under study in order to measure the reflectivity coefficient $\mathrm{R}$ of the neutron beam. In this course, we will only focus on the specular reflectivity that corresponds to the case where the incident and reflected neutron beams are symmetric with respect to the surface normal. This provides the profile of chemical composition perpendicular to the surface, averaged on the whole surface. The principle of off-specular studies that treat the case of neutrons that are scattered out of the specular direction and allow to access to in-plane structural information can be found elsewhere [1].

The lower limit of thickness that can be measured is directly linked to the minimal reflectivity $\mathrm{R}$ that can be experimentally obtained $\left(\mathrm{R} \sim 10^{-6}\right.$ to $10^{-7}$ on samples of a few $\left.\mathrm{cm}^{2}\right)$ with the current brightness of neutrons sources. Such a limit can usually be extended to lower values by X-ray reflectivity, the counterpart experiment of NR with photons of the same wavelengths, and which has thus to be preferred to NR. Nevertheless, NR offers unique

\footnotetext{
* Corresponding author: fabrice.cousin@,cea.fr
} 
advantages that make it irreplaceable in many experimental cases: neutrons can access to buried interfaces, they have a high sensibility for materials containing hydrogen like biological systems and they can probe magnetic properties of materials at the atomic level. This is due to some features of the neutron-matter interaction, as detailed in the following.

First, neutron interacts directly with atom nuclei. The interaction has a very short range and is described by the coherent scattering length $b$. It varies strongly between isotopes of the same chemical element and randomly across the elements of the periodic table, and can be, either positive, or negative. In particular, the $b$ value for hydrogen ${ }^{1} \mathrm{H}$ is negative and has a very different value with respect to all other atoms constitutive of organic molecules $(\mathrm{C}, \mathrm{O}$, $\mathrm{N}, \ldots)$ and importantly of deuterium ${ }^{2} \mathrm{H}$. This enables to achieve experiments on organic materials because the neutron refraction index of a given organic molecule is essentially dependent on its content on hydrogen atoms. Such refraction index can be varied on a large range by replacing some hydrogen atoms by deuterium atoms. Such replacement does not alter most of the physical properties of the molecules, but may change slightly some of them, $e g$ hydrogen bonding. This opens the way to contrast variation experiments where some molecules are labelled with deuterium to create a neutron contrast in the system. It is also possible to continuously tune the neutron refractive index of a solvent in a complex system by mixing hydrogenated and deuterated solvents in order to match one component, making it invisible from the neutron point of view. Such contrast variation experiments have been very successfully applied in soft matter in the field of surfactants and polymers [2-4] or in biophysics [5-7].

Second, neutrons are very weakly absorbed by matter, except for few atoms of the periodic table that have a huge neutron absorption cross-section (for instance boron, gadolinium, or lithium). This weak interaction makes possible the study of buried interfaces or to achieve in situ experiments in various sample environments.

Third, NR experiments are not destructive, contrarily to X-ray experiments, because the energy of neutrons used for experiments is of a few meV.

It is finally worth noting that NR allows the measurement of the magnetization profile in thin magnetic films because neutrons bear a spin $\pm 1 / 2$ and interact with the atoms having a magnetic moment; but this last aspect is out of the scope of this course focused on biology and is documented elsewhere [8].

Such specificities of neutrons have made NR experiments very popular since the early 90 's. But it is important to keep in mind that it is advantageous to combine NR with other surface techniques like X-ray reflectivity, ellipsometry, and with techniques enabling to determine in-plane structure information like either scattering methods (GISAS, surface diffraction, off-specular measurements), or microcopy techniques (AFM, Brewster microscopy).

This course is divided into two parts. In the first one, the principle of NR will be presented. In the second part we will describe how to realize a NR experiment from a practical point of view (description of instruments and of possible geometries of measurements, sample environments, accessibility to neutron beams, neutron facilities...). The treatment and analysis of NR data, as well as examples of application of NR to biological systems, are described in the book section written by Y. Gerelli. 


\section{Principle of Neutron Reflectivity}

The formalism described in this part is a wave formalism very close to the one usually used for reflection of light. It is limited to specular reflectivity and does not consider the absorption of neutrons.

\subsection{Neutron-matter interaction and calculation of refractive index for neutrons}

Let us consider a neutron beam reflecting at an infinite flat surface with an incident angle $\theta$ with respect to the plane $z=0$, as showed in Figure 1 . This surface is defined by the interface between the vacuum $(n=1)$ and a medium of refraction index $n$. In vacuum, the modulus of the wave vector of an incident neutron of wavelength $\lambda$ is $k=2 \pi / \lambda$. In the medium of refractive index $n$, the neutron interacts with the nuclei of atoms. The amplitude of the interaction between a neutron and an atomic nucleus is given by the scattering length $b$, which has the dimension of a length; it may be regarded as a constant for a given isotope. Some typical values of $b$ are given in Table 1 . The neutron-matter interaction is described by the Fermi pseudo-potential [9]; it can be shown that the neutron wave vector $k_{n}$ in a medium of refractive index $n$ can be written:

$$
k_{n}^{2}=k^{2}-4 \pi N b
$$

where $N$ is the number of atoms per volume unit. This last equation allows to express the refractive index $n$ of a homogeneous medium, defined as the ratio of the wave vectors in the material and in vacuum:

$$
n^{2}=\frac{k_{n}^{2}}{k^{2}}=1-\frac{\lambda^{2}}{\pi} N b
$$

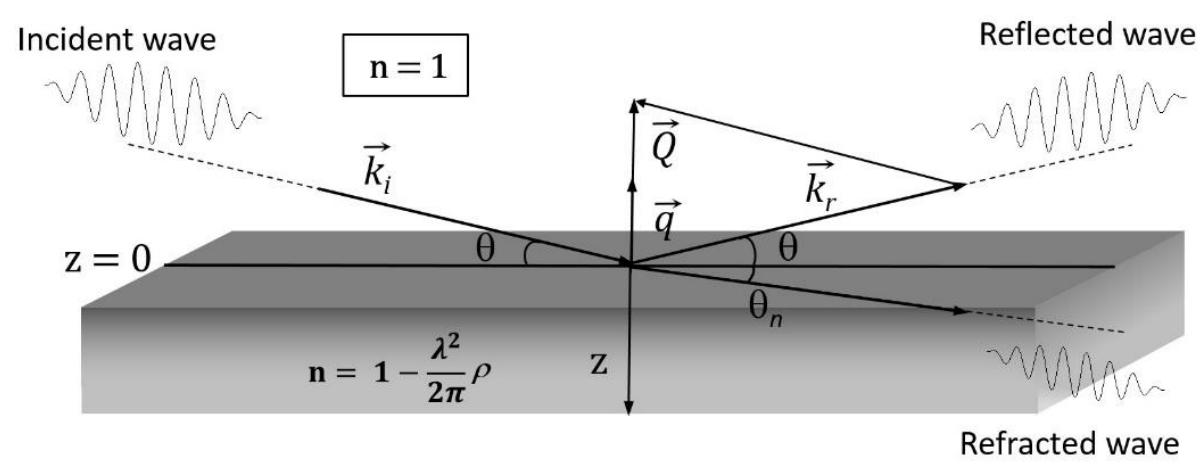

Fig. 1. Reflection on an infinite planar surface.

The product $N b$ is the neutron coherent scattering length density (SLD) $\rho$. Table 1 shows the coherent scattering length $b$ of some atoms and the coherent scattering length density $\rho$ of some materials [10]. Some full neutron tables of elements can be easily found on internet [11]. The SLD of the substrate is a crucial information in a NR experiment. It has to be ideally calculated prior to the experiment. Its calculation is very simple for crystals or 
solvents $^{\dagger}$ but may raise difficulties for molecules in solution when their specific volumes are not known with high precision.

The characteristic wavelengths of the incident neutrons being always of the order of few $\AA$ and the SLD of the order of $10^{-6} \AA^{-2}$, the values of the neutron refractive index are always very close to $1\left(\sim 1 \pm 10^{-3}-10^{-4}\right)$. Therefore, unlike the visible light, reflection/refraction for neutrons can only occur at very grazing angles, of the order of $\sim 1^{\circ}$ and the refractive index $n$ for neutrons can be larger than 1 .

Since $n$ is very close to 1 , a series expansion of eq. (2), enables to write:

$$
n \approx 1-\frac{\lambda^{2}}{2 \pi} \rho
$$

Table 1. Coherent scattering length of some atoms and scattering length density of some molecules. PC stands for phosphocholine.

\begin{tabular}{|c|c|c|c|c|c|}
\hline \multicolumn{7}{|c|}{ Coherent scattering length $b\left(10^{-12} \mathrm{~cm}\right)$} \\
\hline${ }^{1} \mathrm{H}$ & $\mathrm{D}\left({ }^{2} \mathrm{H}\right)$ & $\mathrm{C}$ & $\mathrm{O}$ & $\mathrm{N}$ & $\mathrm{Si}$ \\
\hline-0.374 & 0.667 & 0.665 & 0.580 & 0.936 & 0.415 \\
\hline \multicolumn{7}{|c|}{ Scattering Length Density $\rho\left(10^{-6} \AA^{-2}\right)$} \\
\hline $\mathrm{H}_{2} \mathrm{O}$ & $\mathrm{D}_{2} \mathrm{O}$ & $\mathrm{PC}_{\mathrm{H}}$ & $\mathrm{PC}_{\mathrm{D}}$ & $\mathrm{SiO}_{2}$ & $\mathrm{Si}$ (crystal) \\
\hline-0.56 & 6.38 & -0.38 & 7.1 & 3.41 & 2.07 \\
\hline
\end{tabular}

Analogously to the geometrical optics, refraction of neutrons at an interface between the vacuum and a medium of index $n$ is described by the Snell-Descartes law:

$$
\cos \theta=n \cos \theta_{n}
$$

where the angles $\theta$ and $\theta_{n}$ are depicted in Figure 1 . When $\theta_{n}=0$, we can define the critical angle for total reflection $\theta_{c}$ :

$$
\cos \theta_{c}=n
$$

Neutrons are totally reflected for $\theta \leq \theta_{c}$. If $n$ is higher than 1 , the conditions of total reflection are never fulfilled, which happens for example at the air/water interface when the substrate is $100 \% \mathrm{H}_{2} \mathrm{O}$. From equations (2) and (5), we obtain:

\footnotetext{
${ }^{\dagger}$ Calculation of SLD in the case of water. Since the molar mass of $\mathrm{H}_{2} \mathrm{O}$ is $18 \mathrm{~g} / \mathrm{mol}$ and the density of $\mathrm{H}_{2} \mathrm{O}$ is $1 \mathrm{~g} / \mathrm{cm}^{3}$, the volume of a molecule $V$ of water is $18 / 6.0210^{23}=2.9910^{-23} \mathrm{~cm}^{3}$ per molecule, for both $\mathrm{H}_{2} \mathrm{O}$ and $\mathrm{D}_{2} \mathrm{O}$. Since $\rho=\left(\Sigma_{\mathrm{i}} b_{\mathrm{i}}\right) / V$ where the $b_{i}$ correspond to the atoms constitutive of the water molecule (table 1), one obtains:

$\rho\left(\mathrm{H}_{2} \mathrm{O}\right)=(-0.374 * 2+0.58) \cdot 10^{-12} / 2.9910^{-23}=-0.5610^{10} \mathrm{~cm}^{-2}\left(-0.5610^{-6} \AA^{-2}\right)$

$\rho\left(\mathrm{D}_{2} \mathrm{O}\right)=(0.667 * 2+0.58) .10^{-12} / 2.9910^{-23}=6.3810^{10} \mathrm{~cm}^{-2}\left(6.3810-6 \AA^{-2}\right)$
} 


$$
\sin \theta_{c}=\sqrt{\frac{\rho}{\pi}} \lambda
$$

When total reflection occurs, the measurement of the critical angle $\theta_{\mathrm{c}}$ for a given wavelength is a very precise measurement of the substrate SLD and therefore of its chemical composition.

\subsection{Reflection on a succession of layers}

The reflectivity is a function that only depends on the scattering vector $\boldsymbol{Q}$ defined by:

$$
\begin{gathered}
\vec{Q}=\overrightarrow{k_{r}}-\overrightarrow{k_{i}} \\
Q=\left\|\overrightarrow{k_{r}}-\overrightarrow{k_{i}}\right\|=\frac{4 \pi \sin \theta}{\lambda}
\end{gathered}
$$

It is equal to twice the projection of the incident wave vector $\boldsymbol{k}_{\boldsymbol{r}}$ on the $z$ axis perpendicular to the surface, as depicted in Figure 1. In the following we will use in calculations the variable $q$, projection of the wave vector on $z$ such that $\boldsymbol{q}=\boldsymbol{Q} / 2$.

If the system under study is made of several layers, each of them having a refractive index $n_{p}$, neutrons will be partially reflected and transmitted from the layer $p$ to the $p+1$ one. From equation (1) the scattering wave vector for each layer writes:

$$
\begin{aligned}
& q_{p}^{2}=q^{2}-4 \pi \rho_{p} \\
& q_{p+1}^{2}=q^{2}-4 \pi \rho_{p+1}
\end{aligned}
$$

The continuity conditions for the wave functions and its derivative at the interface $(p / p+1)$ lead us to write the reflection coefficient $\mathrm{R}$, which is defined as the ratio of the intensity of the beam reflected by the layer $p+1$ to the intensity of the incident beam on such a layer:

$$
R=\frac{\left|B_{p}\right|^{2}}{\left|A_{p}\right|^{2}}=\left|\frac{q_{p}-q_{p+1}}{q_{p}+q_{p+1}}\right|^{2}
$$

where $A_{p}$ and $B_{\mathrm{p}}$ are respectively the amplitudes of the wave propagating towards the inner and the outer of the material.

Using this equation recursively it is possible to calculate the whole reflectivity of the system. This method is known as the optical matrix method and uses the Abélès formalism [12].

It is easy to show that reflectivity from a simple single interface between two media of refractive index $n_{0}$ and $n_{l}$, respectively, can also be written:

$$
R=\left|\frac{n_{0} \sin \theta_{0}-n_{1} \sin \theta_{1}}{n_{0} \sin \theta_{0}+n_{1} \sin \theta_{1}}\right|^{2}
$$

from Snell-Descartes condition we can write:

$$
n_{1} \sin \theta_{1}=\left(n_{1}^{2}-n_{0}^{2} \cos ^{2} \theta_{0}\right)^{1 / 2}
$$




\subsection{The ideal interface and the Fresnel reflectivity curve}

Let us consider here a neutron beam propagating in vacuum (or air) and reflecting on an ideal planar substrate without any layer at the surface. If the interface is perfect with no roughness, such a system is a diopter. The reflectivity of such an interface is called the Fresnel reflectivity $\left(R_{F}\right)$. The Fresnel reflectivity depends on the SLD of the substrate $\rho_{s}$ and writes:

$$
R_{F}=\left|\frac{B}{A}\right|^{2}=\left|\frac{q-q_{s}}{q+q_{s}}\right|^{2}=\left|\frac{1-\left(1-\left(\frac{q_{c}}{q}\right)^{2}\right)^{1 / 2}}{1+\left(1-\left(\frac{q_{c}}{q}\right)^{2}\right)^{1 / 2}}\right|^{2}
$$

where $q_{c}$ and $q_{s}$ are obtained from:

$$
\begin{aligned}
q_{c}^{2} & =4 \pi \rho_{s} \\
q_{s}^{2} & =q^{2}-4 \pi \rho_{s}=q^{2}-q_{c}^{2}
\end{aligned}
$$

$\boldsymbol{q}_{c}$ is the critical scattering vector that separates total reflection that occurs below $q_{c}$, from partial reflection where a part of the beam is refracted (above $q_{c}$ ). At $q_{c}$, the wave propagates only at the surface and parallel to it. Far from the critical angle $\left(q>>q_{c}\right), R_{F} \propto(1 / q)^{4}$. This means that the decay in reflectivity is always very fast whatever the layer at the surface. The NR curves $R=\mathrm{f}(q)$ are thus traditionally represented in logarithmic-linear scales or logarithmic-logarithmic scales (Figure 2). Another convenient way to represent $R_{F}$ is the Fresnel representation $f(q)=\mathrm{R}(q) q^{4}$ for which the $q^{4}$ term compensates the intrinsic $q^{-4}$ decay of the NR curve to highlight the features coming from the layers at the interface.

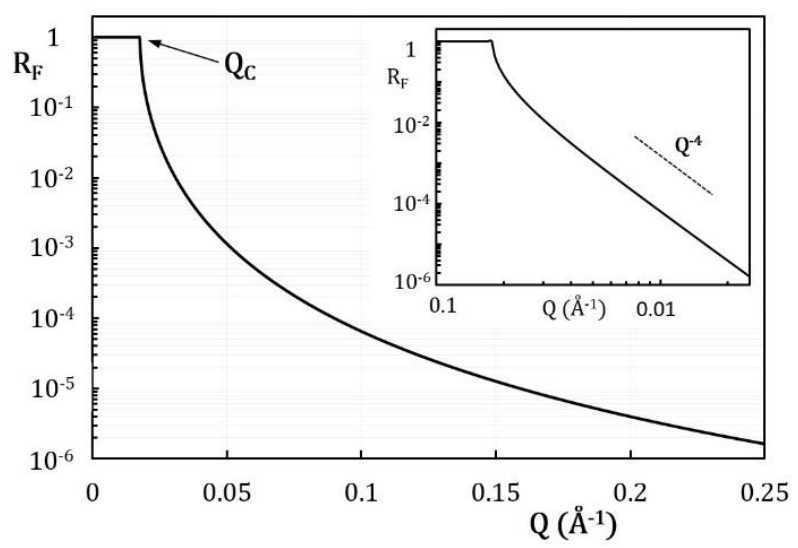

Fig. 2. Logarithmic-linear representation and logarithmic-logarithmic representation (inset) of the Fresnel reflectivity as a function of $Q$ for $\rho_{\mathrm{s}}=6.3810^{-6} \AA^{-2}$ (air/ $\mathrm{D}_{2} \mathrm{O}$ interface). The SLD of air $\rho_{\text {air }}$ is considered as equal to 0 owing to the very low density of the gas.

\subsection{The case of a homogeneous layer on a substrate}

Let us consider a single homogeneous layer of thickness $d$ deposited on the surface of a perfectly flat substrate of thickness that can be considered infinite (Figure 3). Such a system is composed of three media: the air (of refractive index 1 and scattering length density 0 ), the layer (of refractive index $n_{1}$ and scattering length density $\rho_{1}$ ) and the substrate (of refractive index $n_{\mathrm{s}}$ and scattering length density $\rho_{\mathrm{s}}$ ) and of two interfaces. Let us set the air/layer 
interface at $z=0$ and the layer/substrate interface at $z=d$. An incident neutron beam coming from the air will be partially reflected at the first interface $(z=0)$ and partially transmitted (for $q>q_{\mathrm{c}}$ ). The transmitted wave will then be partially reflected and partially transmitted at the layer substrate interface $(z=d)$. The two reflected waves will interfere constructively or destructively depending on their phase difference which is related to their difference on optical path and thus to the thickness of the layer. It therefore appears qualitatively that the reflectivity curve will exhibit interference fringes that enable the measurement of the layer thickness.

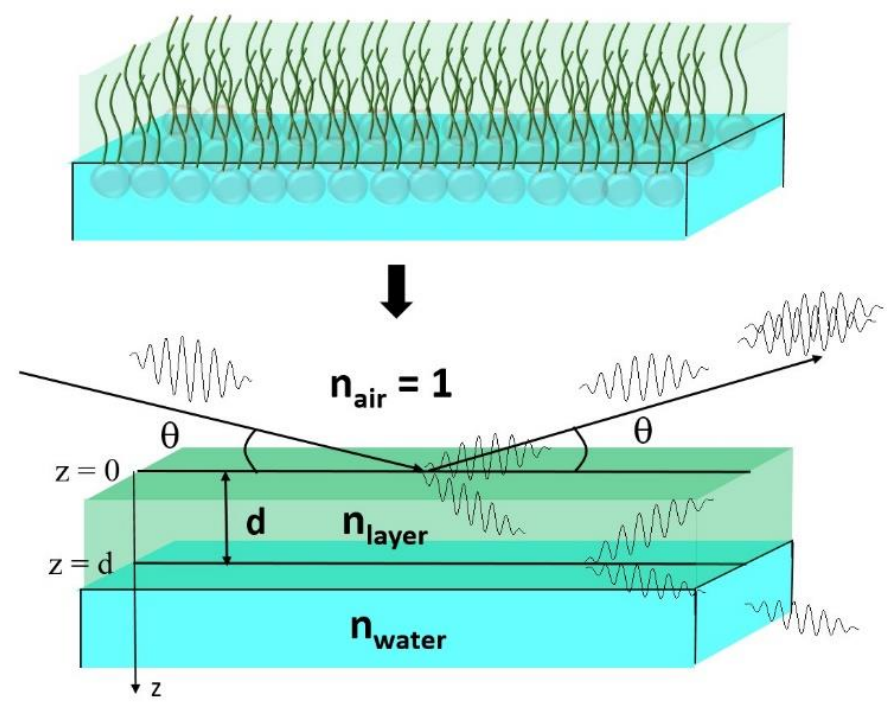

Fig. 3. (Color online). Reflection of a homogeneous monolayer of phospholipids at the air/water interface. The hydrophobic tails form a thin film of homogeneous SLD and thickness $d$. The water is semi-infinite substrate. The phospholipids heads are not considered here.

Figure 4 presents some typical NR curves for monolayers of different thicknesses and SLD at the air/water interface. R exhibits typical oscillations that are called the Kiessig fringes. The fringe spacing is $\Delta \boldsymbol{q}=\mathbf{2} \pi / \boldsymbol{d}$. This is the Bragg relationship corrected from the critical angle:

where $m$ is an integer.

$$
2 d \sqrt{\sin \theta^{2}-\sin \theta_{c}^{2}}=m \lambda
$$

The inter-fringes distance is linked to the thickness of the layer and their amplitude comes from the difference of SLD between the monolayer and both air and substrate. 


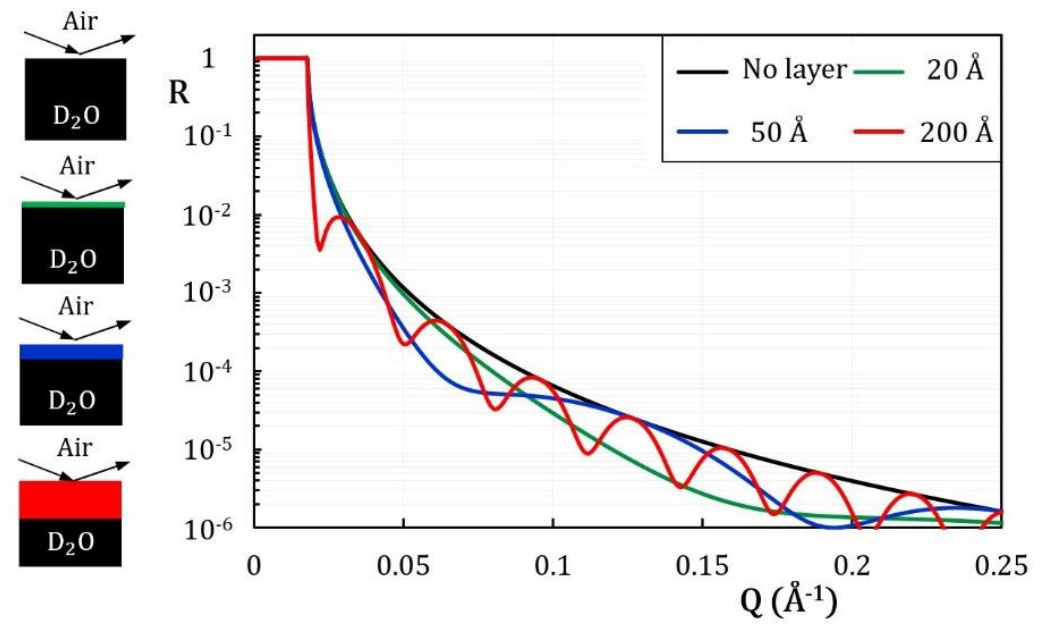

Fig. 4a. (Color online). Reflectivity $\mathrm{R}$ calculated for a single layer at the air $/ \mathrm{D}_{2} \mathrm{O}$ interface of different thicknesses $d$ and constant player $\left(4.610^{-6} \AA^{-2}\right)$. The sketches in the left panel are schematic representations of the system.

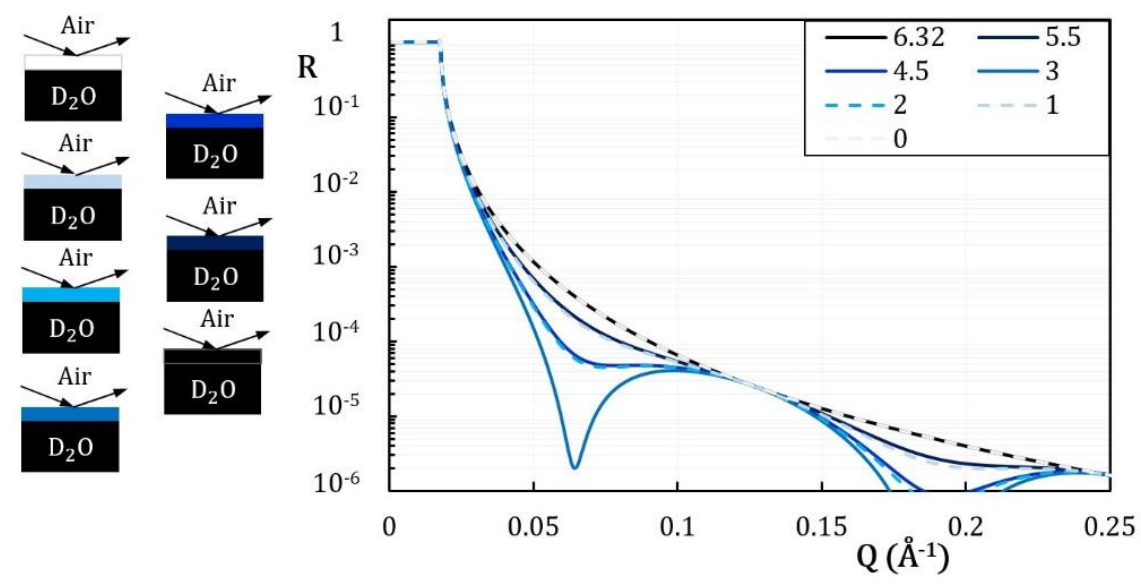

Fig. 4b. (Color online). Reflectivity $\mathrm{R}$ calculated for a single layer at the air/ $\mathrm{D}_{2} \mathrm{O}$ interface for different

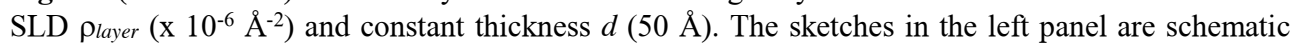
representations of the system.

\subsection{Roughness and inter-diffusion}

In the previous parts, we have considered that the interface between the layer $p$ and the layer $p+1$ was perfect with no heterogeneities, which means that there is a sharp discontinuity of SLD when passing from $\rho_{\mathrm{p}}$ to $\rho_{\mathrm{p}+1}$ (step function). This is only theoretical; in practice, interfaces are never so abrupt because of two different physical phenomena: $(i)$ roughness and/or (ii) inter-diffusion (see Figure 5). Roughness is due to the fact that the surface is irregular, as shown in Figure 5.a. and is equivalent to the case where neutrons are reflected by many surfaces with different angular orientations and a significant part of them is out of 
the specular plane. Inter-diffusion occurs when two successive layers of different composition diffuse slightly one into each other, creating a smooth transition (Figure 5.b).

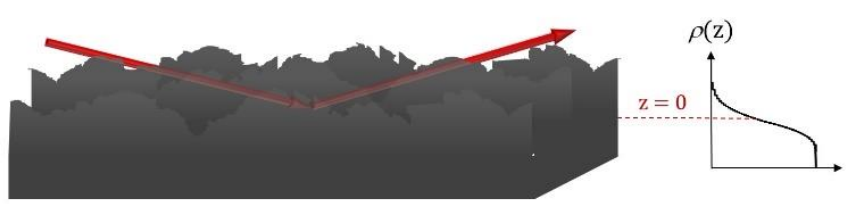

(a)

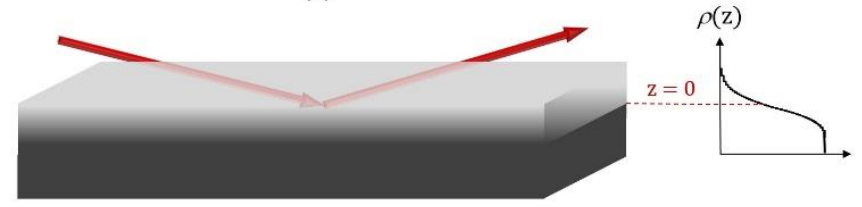

(b)

Fig. 5. (a) Schematic representation of roughness; (b) schematic representation of inter-diffusion. The profiles represented on the right show the effective $\rho(z)$ profile averaged on the whole surface.

Assuming that both kinds of interfaces have a Gaussian profile, they will be simulated by the same error function, where $p / p+1$ is the interface between two successive layers:

$$
\operatorname{erf}\left(\frac{z-z_{p / p+1}}{\sigma_{p / p+1}}\right)=\frac{2}{\sqrt{\pi}} \int_{0}^{(z-z p / p+1) / \sigma_{p / p+1}} e^{-t^{2}} d t
$$

This function has an inflection point at $z_{\mathrm{p} / \mathrm{p}+1} . \sigma_{\mathrm{p} / \mathrm{p}+1}$ is the inverse of the tangent slope of the curve at $z_{\mathrm{p} / \mathrm{p}+1}$. The thickness of the interface is given by $2 \sigma_{\mathrm{p} / \mathrm{p}+1}$. Another method to take into account roughness is to discretize the density profile in a large number of layers having a low variation of $\rho$ from one layer to the next. However, the calculation time may become very long.

Figure 6 shows the effect of roughness/interdiffusion on the NR curve calculated in the case of a single layer at the air/water interface (same parameters as in Figure 4).

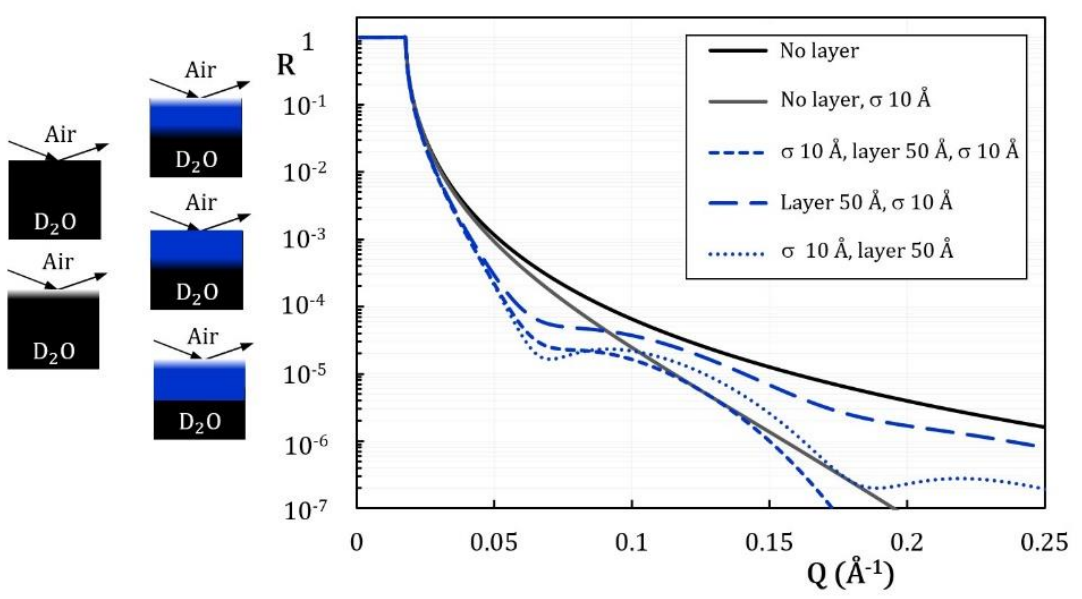

Fig. 6. (Color online). Influence of the roughness $(\sigma=10 \AA)$ on the reflectivity R calculated for a single layer $\left(\rho_{\mathrm{s}}=4.610^{-6} \AA^{-2}, d=50 \AA\right)$. The sketches in the left panel are schematic representations of the system. 


\section{Practical aspects}

This part is devoted to the experimental aspects of NR to help users achieving successful experiments.

\subsection{Instruments}

\subsubsection{Principle of measurement}

In neutron sources, whether neutrons are produced with a continuous flux in a nuclear reactor or by pulses in a spallation source, the energy of the produced neutrons is of the order of a few $\mathrm{MeV}$; they must, therefore, be thermalized in order to perform neutron scattering experiments. The neutron energies needed for NR are of a few meV (cold neutrons) with corresponding wavelengths ranging between $2 \AA$ and $20 \AA$. The thermalized neutron beam is always polychromatic.

It exists two ways to measure the reflectivity of a sample as a function of the scattering vector $Q=4 \pi \sin \theta / \lambda$ : either by varying $\theta$ at fixed $\lambda$, or by varying $\lambda$ at fixed $\theta$.

In the first case, called $\boldsymbol{\theta}-\mathbf{2} \boldsymbol{\theta}$ mode, a monochromator, e.g. a mono-crystal, is used to select the needed wavelength $\lambda$; the neutron beam is then collimated using collimation slits and sent to the sample at grazing incidence. One then measures the reflected beam, step by step, by rotating the sample of an angle $\theta$ and the detector of an angle $2 \theta$. The illumination of the sample varies from one angle to the other, which has to be taken into account in data reduction.

In the second case, called time-of-flight mode, one exploits the fact that neutrons travel at a finite speed $v$ that is rather slow as it lies between a few $100 \mathrm{~m} / \mathrm{s}$ and a few $1000 \mathrm{~m} / \mathrm{s}$ for cold neutrons. Neutron speed $v$ is related to wavelength $\lambda$ through the de Broglie relationship $v=h / m \lambda$, where $h$ is the Planck constant and $m$ the neutron mass. In a time of flight experiment, a pulsed white beam is sent to the sample at a fixed angle $\theta$ : it is possible to separate neutrons of different wavelengths through their speed, the fastest neutrons arriving on the detector before the slower ones. Pulses are spontaneously generated in a spallation source but have to be created by a chopper, a rotating disk containing neutron-transparent windows, in a reactor source. The collimated neutron beam is sent at grazing incidence on the sample; if one defines the origin of times $t_{0}=0$ the time at which the chopper generates a pulse, the wavelength of neutrons arriving at the detector, placed at a known distance $\mathrm{L}$, is determined by the measurement of the time $t$ taken to every neutron to flight from the chopper to the detector, since $\lambda=t h / m L$. It is assumed that the speed of neutrons does not change when they interact with the samples (elastic scattering). Measuring the coefficient of reflection is simple: the white incident polychromatic beam is first measured directly on the detector without any reflection and the measured reflected beam is then divided by such incident white beam for every $\lambda$ value.

For biological and soft matter studies, the dedicated reflectometers work with the timeof-flight mode because it has incomparable advantages with respect to the $\theta-2 \theta$ mode: $(i)$ working at constant $\theta$ does not force to rotate the sample, which is highly appreciable for measurements at the air/water interface; (ii) all the points of a reflectivity curve are measured at the same time, which removes any incertitude on the possible ageing of the sample during measurement; (iii) kinetic measurements are possible.

In a NR experiment, the incident angle on the surface under study is always very small, ranging typically from $0.5^{\circ}$ to $5^{\circ}$. Measurements at lower angles are indeed practically almost impossible because the reflected beam has to be separated from the incident non-deviated direct beam. The range of scattering vectors lies then between $0.003 \AA^{-1}$ and $0.3 \AA^{-1}$ for cold 
neutrons. This fixes the typical range of thickness that can be measured from around $5 \AA$ to $2000 \AA$. The collimation is achieved by a simple set of rectangular slits made of neutron absorbers of various apertures. The sample is mounted on a goniometer in order to set the desired angle. In the experiments at the air/water interface where it is not possible to rotate the sample, the neutron beam is deviated using a neutron mirror upstream of the sample in order to obtain the desired angle at the air/water measurements. A typical sketch of a timeof-flight reflectometer at the air/water interface is depicted in Figure 7.

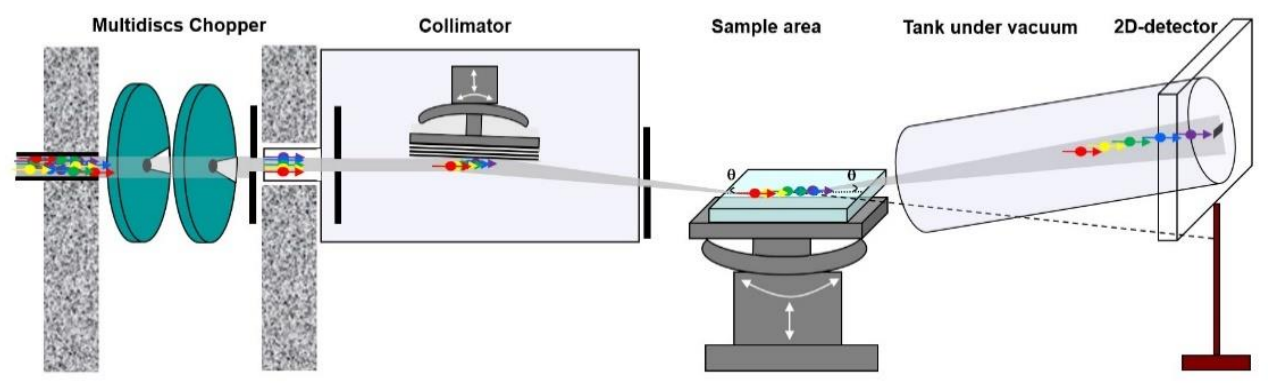

Fig. 7. (Color online). Typical sketch of a time-of-flight reflectometer on a continuous neutron source (reactor). A double-chopper located in the casemate creates pulses of polychromatic neutrons from the continuous beam. The chopped beam is then collimated by slits and can be deviated by a supermirror if necessary for measurements at the air/water interface. The reflection of neutrons on the sample is considered as elastic. The determination of the speed, i.e. of the wavelength, is done by measuring the time of neutron flight from the chopper to the detector. Neutrons of different wavelengths are depicted by arrows of different colors.

The typical acquisition time depends on the requested minimal reflectivity $R$. If $R$ is of the order of $10^{-5}$, a few minutes are enough to obtain a correct statistics on state-of-the art instruments with a sample of $\sim 10 \mathrm{~cm}^{2}$ (see next section), but this time has to be extended up to a few hours to reach $10^{-6}$ or below.

\subsubsection{Experimental resolution}

The major weakness of NR is the poor flux of the neutron beam provided by the actual sources, whether reactor or spallation sources, even on the most powerful ones. In order to make a proper measurement with a very good signal-to-noise ratio, a compromise has to be done between flux and resolution. In biological or soft matter studies, performing the experiments in degraded resolution is not an issue since the probed interfaces are soft. The usual chosen $\delta q / q$ resolution lies thus generally between $5 \%$ and $10 \%$. This resolution originates both from the $\delta \theta / \theta$ of the collimation and from the $\delta \lambda / \lambda$ of the chopper (or a monocrystal or a mechanical selector in the case of a reflectometer working in $\theta-2 \theta$ mode with a monochromatic beam).

$$
\left(\frac{\delta q}{q}\right)^{2}=\left(\frac{\delta \theta}{\theta}\right)^{2}+\left(\frac{\delta \lambda}{\lambda}\right)^{2}
$$

The divergence of the incident beam $\delta \theta$ and the angular resolution $\delta \theta / \theta$ are set by the apertures of the entrance and exit slits of the collimator, which are opened usually at around 1 or few $\mathrm{mm}$, and by the collimator length, which is usually a few meter long. For an entrance slit of aperture $S_{\text {ent }}$, an exit slit of aperture $S_{\text {exit }}$ and a collimator length $D_{c}$, the divergence is tan $\delta \theta=\left(S_{\text {ent }}+S_{\text {exit }}\right) / 2 D_{c}$; it can be taken into account in the calculation. The expressions for 
reflectivity presented in the precedent part have been calculated for an incident beam without divergence. The calculation of the $\delta \lambda / \lambda$ of a chopper is much trickier and is detailed in [13].

\subsection{Samples and possible interfaces that can be probed}

The geometry of a NR experiment imposes three imperative conditions that have to be absolutely fulfilled to make it feasible. First, the medium in which the neutron beam is travelling upstream the interface and after reflection has to be totally or partially transparent to neutrons, otherwise the signal is lost. Second, the flatness of the studied interface has to be as best as possible: if the interface has a curvature, this would have an effect equivalent to an increase of the divergence $\delta \theta$ of the beam. Although this divergence could be taken into account by an increase of the effective $\delta \theta$ within the resolution function during the data modelling, it has to be as limited as possible. This imposes some limitations on the different possible geometries of measurements and on the samples themselves. Third, the roughness of the surface has to be very limited, otherwise it would induce a very steep drop of reflectivity that leads to a loss of information on the SLD profile.

\subsubsection{The different possible geometries of measurements}

In biology, almost all NR studies focus on processes that occur between biological species (proteins, viruses, peptides, ...) and phospholipids bilayers (membranes) or monolayers. The most interesting interfaces are then liquid/liquid interfaces and air/water interfaces. However, there are only very sparse studies probing true liquid/water interfaces by NR since this is something hardly challenging for some reasons that are detailed elsewhere [14]. Thus, in practice, liquid/water interfaces are replaced by experiments at solid/water interfaces where membranes are deposited on solid substrates, as depicted in the book section written by Gerelli.

Experiments at the air/water interface are very easy to implement since air is almost transparent to neutrons and therefore the attenuation of the neutron beam on a few $\mathrm{cm}$ in air is negligible.

Conversely, in NR experiment at the solid/water interface, the transparency of media in which the neutron beam has to travel is a key point to consider. In practice, two processes attenuate the beam: absorption and incoherent scattering. In biological studies, neutron absorption is never an issue because water and biological compounds contain none of the few elements of the periodic table that have an important absorption cross-section for neutrons $\left({ }^{3} \mathrm{He},{ }^{6} \mathrm{Li}, \mathrm{B}, \mathrm{Gd}, \ldots\right)$ [11]. However, the beam transmission can also be reduced due to incoherent scattering, that occurs with a constant probability over $4 \pi$ independently from the position of scatterers [9], when this latter is important. Indeed, although the incoherent scattering cross-section is null or very weak for most elements of the periodic table, it is unfortunately very high for hydrogen ${ }^{1} \mathrm{H}$ and important for ${ }^{2} \mathrm{H}$ deuterium. For example, the transmission of a neutron beam is only 0.5 through $1 \mathrm{~mm}$ of $\mathrm{H}_{2} \mathrm{O}$ and 0.8 through $2 \mathrm{~mm}$ of $\mathrm{D}_{2} \mathrm{O}$ for $6 \AA$ neutrons. Therefore, since in a NR experiment the beam has an incident grazing angle (less than $5^{\circ}$ ), the neutron beam has to travel for several centimeters within the sample; in this case a particular geometry has to be used in order to avoid crossing water, as shown in Fig.8.c. The neutron beam has thus to pass through the solid support that has to be made with a material transparent to neutrons.

\subsubsection{Samples and samples environments}


The surface of the sample has to be optimized. Since the raw number of reflected neutrons is directly proportional to the surface of illumination of the sample by the neutron beam prior to the data reduction step, the surface of the sample has to be maximized in order to get a good signal-to-noise ratio with reasonable acquisition times. With the current performances of the state-of-the-art reflectometers, the order of magnitude of the sample surface allowing good measurements is a few $\mathrm{cm}^{2}$. It must be avoided to decrease surfaces area lower than $1 \mathrm{~cm}^{2}$. Moreover, at the air/water interface, it is mandatory that the sample surface is sufficiently large so that the beam illuminates a perfectly flat area of the surface, thus located away enough from the meniscus at the edges of the trough containing the water.

At the solid/liquid interface, the solid substrate to be crossed by neutrons must be transparent to neutrons, must have a very small (root mean square ( $r m s$ ) roughness and must be rigid enough not to deform mechanically under internal stresses, so that it does not create a curvature at the interface. In practice, the only solid substrates that are used in NR for biological studies are silicon wafers (Fig. 8.d). Sapphire blocks or quartz crystals have also been seldom used in soft matter but they are much more expensive. Silicon wafers are indeed nearly transparent to neutrons because their incoherent scattering cross-section is null and their absorption cross-section is very weak. They can easily be purchased with an excellent mosaicity ( $r m s$ roughness of a few $\AA$ ) at low cost from silicon manufacturers. They are always covered by a very thin layer of $\mathrm{SiO}_{2}(\sim 10$ to $20 \AA)$ at their surface. Such silica layer may pose problems during data fitting but they can also offer an advantage since $\mathrm{Si}-\mathrm{OH}$ groups enable many potential chemical modifications in order to render the surface hydrophilic, hydrophobic, or electrically charged, for example.

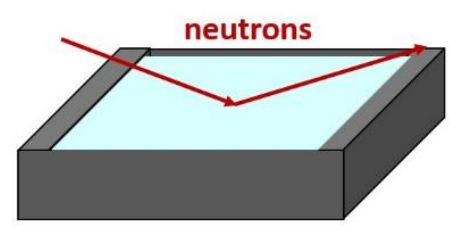

(a)

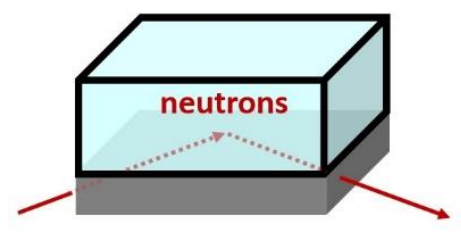

(c)

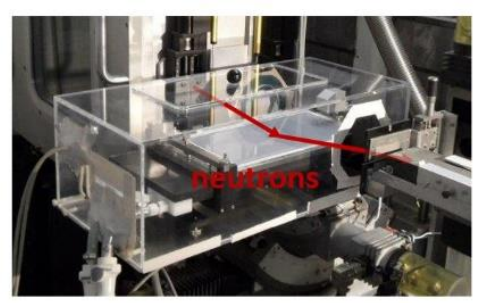

(b)

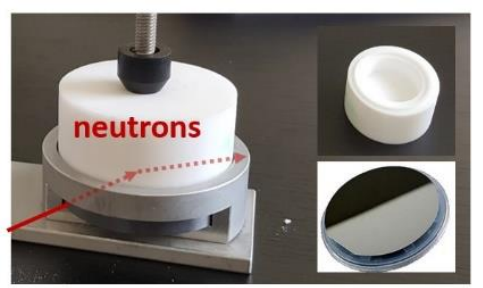

(d)

Fig. 8. (Color online). (a) Standard measurement geometry at the air/water interface. (b) Langmuir trough installed on the EROS reflectometer (LLB, France) [15] for measurements at air/water interface. (c) Standard measurement geometry at the solid/water interface. (d) Typical cell for measurement at solid/water interface; insets show respectively the reservoir for water (upper picture) and the silicon wafer with its polished surface (lower picture).

Thanks to the fact that neutrons are poorly absorbed by almost all atoms of the periodic table, it is easy to design various sample environments for in situ experiments. At the air/water interface, the most used equipment is the Langmuir trough (Fig. 8.b) since it enables to set the concentration of the monolayer under study at the targeted surface pressure. At the solid/water interface, there are various measurements cells in the different neutron facilities, 
that can be equipped with various ancillary options like solvent circulation baths, temperature controllers or electrochemical cells for electric measurements [16]; an example of the application of an electrochemical cell to lipids is presented in [17]. When filling such cells, it has to be avoided that air bubbles locate at the silicon/water interface, which is easily achieved on horizontal reflectometers when the water is placed above the silicon (Fig. 8.d).

\subsection{Access to instruments}

Reflectometers are accessible to the scientific community in large neutron facilities. The beamtime (e.g. 2 to 5 days) is allocated after acceptation of proposal experiments after reviewing by a committee of experts. Call for proposals takes place generally twice a year. Fast access or test experiments are also sometimes possible and have to be negotiated with facilities. The actual landscape of European neutron facilities has strongly changed these last years with the closure of Orphée reactor at Saclay (France) and HMI at Berlin (Germany). State-of-the-art reflectometers can now only be accessed in Europe at ISIS (United Kindgom), MLZ (Germany), PSI (Switzerland) and at ILL, the European source located in Grenoble, France [18]. Two reflectometers are especially suited to biological studies at ILL, namely FIGARO, a horizontal reflectometer dedicated to soft matter and biophysics, and D17, a vertical reflectometer for multipurpose measurements. In the near future, the new European Spallation Source (ESS, Lund, Sweden) will be operating and FREIA, a high flux horizontal reflectometer, is forecast for 2025 in the instrumental suite [19].

The authors thank the organizers of the "Neutrons and Biology School 2018" of the French Neutron Society for their kind invitation to participate to this special issue. They also thank $\mathrm{D}^{\mathrm{r}}$ Louis Bondaz whose $P h D$ manuscript has inspired Figures 2, 4, and 6 of this course.

\section{References}

1. M. Wolff, EPJ Web Conf., 188, 04002 (2018)

2. J. Penfold, R. K. Thomas, J. Phys. Condens. Matter, 2(6), 1369-1412 (1990).

3. T. P. Russell, Mater. Sci. Reports, 5, 171-271, (1990)

4. L. Braun, M. Uhlig, R. von Klitzing, R. A. Campbell, “Adv. Colloid Interface Sci., 247, 130-148 (2017)

5. H. P. Wacklin, Curr. Opin. Colloid Interface Sci., 15, 445-454, (2010)

6. G. Fragneto, Eur. Phys. J. Spec. Top., 213, 327-342 (2012)

7. G. Fragneto-Cusani, J. Phys. Condens. Matter, 13(21), 4973-4989 (2001)

8. F. Ott, École thématique la Société Française la Neutronique, 13, 02004 (2014)

9. G. L. Squires, Introduction to the Theory of Thermal Neutron Scattering, (Cambridge: Cambridge University Press, 1978)

10. V. Sears, Neutron news, 3, 26-37 (1992)

11. https://www.nist.gov/ncnr/neutron-scattering-lengths-list.

12. L. G. Parratt, Phys. Rev., 95(2), 359-369 (1954)

13. D. Lairez, A. Chennevière, F. Ott, J. Appl. Cryst, 53, 464-476 (2020)

14. F. Cousin, A. Chennevière, EPJ Web Conf., 188, 04001 (2018)

15. F. Cousin, F. Ott, F. Gibert, A. Menelle, Eur. Phys. J. Plus, 126, 1-11 (2011)

16. A. Koutsioubas, D. Lairez, G. Zalczer, F. Cousin, Soft Matter, 8, 2638-2643 (2012)

17. G.C. Fadda, D. Lairez, Z. Guennouni, A. Koutsioubas, Physical Review Letters, 111(2), 
028102 (2013)

18. https://www.ill.eu/

19. https://europeanspallationsource.se/ 